\title{
Asymptotic Giant Branch predictions: Theoretical uncertainties
}

\author{
S. Cassisi ${ }^{1}$, V. Castellani ${ }^{2,3}$, S. Degl'Innocenti ${ }^{2,3}$, G. Piotto $^{4}$, and M. Salaris ${ }^{5}$ \\ 1 Osservatorio Astronomico di Collurania, via M. Maggini, 64100 Teramo, Italy \\ 2 Dipartimento di Fisica, Universitá di Pisa, Piazza Torricelli 2, 56126 Pisa, Italy \\ 3 Istituto Nazionale di Fisica Nucleare, Sezione di Pisa, via Livornese 582/A, S. Piero a Grado, 56010 Pisa, Italy \\ 4 Dipartimento di Astronomia, Universitá di Padova, Vicolo dell'Osservatorio 5, 35122 Padova, Italy \\ ${ }_{5}$ Astrophysics Research Institute, Liverpool John Moores University, Twelve Quays House, Egerton Wharf, \\ Birkenhead L41 1LD, UK
}

Received 27 June 2000 / Accepted 9 November 2000

\begin{abstract}
In this paper we investigate the level of agreement between observations and "new" Asymptotic Giant Branch (AGB) models, as produced by updating the physical inputs adopted in previous stellar computations. One finds that the new physics increases the predicted luminosity of Horizontal Branch (HB) and AGB stellar structures by a similar amount, keeping unchanged the predictions about the difference in luminosity between these two evolutionary phases. The best fit of selected globular clusters appears rather satisfactory, disclosing the relevance of the assumption on the mass of the Red Giant Branch (RGB) progenitor in assessing the distance modulus of moderately metal rich clusters. The still existing uncertainties related either to the input physics or to the efficiency of some macroscopic mechanisms, like convection or microscopic diffusion, are critically discussed, ruling out the occurrence of the so called "breathing pulses" during the central He exhaustion, in agreement with earlier suggestions.
\end{abstract}

Key words. stars: AGB - stars: evolution - stars: Hertzsprung-Russel diagram

\section{Introduction}

The capability of current stellar models to account for all the evolutionary phases observed in stellar clusters is undoubtedly an exciting achievement which crowns with success the development of stellar evolutionary theories as pursued all along the second half of the last century. Following such a success, one is often tempted to use evolutionary results in an uncritical way, i.e., taking these results at their face values without allowing for theoretical uncertainties. However, theoretical uncertainties do exist, as it is clearly shown by the not negligible differences still existing among evolutionary results provided by different theoretical groups.

The discussion of these theoretical uncertainties was early addressed by Chaboyer (1995) in a pioneering paper investigating the reliability of theoretical predictions concerning H-burning structures presently evolving in galactic globular clusters (GCs) and, in turn, on the accuracy of current predictions about GC ages. More recently, such an investigation has been extended to later

Send offprint requests to: S. Degl'Innocenti, e-mail: scilla@astr18pi.difi.unipi.it phases of stellar evolution by Cassisi et al. (1998, hereinafter CCDW; 1999), and Castellani \& Degl'Innocenti (1999), who discussed theoretical predictions concerning central He-burning low-mass stars populating the Horizontal Branch of galactic globular clusters.

In this paper we will discuss predictions concerning the evolutionary behaviour of Asymptotic Giant Branch stars, devoting particular attention to two key observational parameters, such as the luminosity of the predicted AGB clump and the number ratio between HB and AGB stars $N_{\mathrm{AGB}} / N_{\mathrm{HB}}$. These parameters appear of particular relevance since the AGB clump luminosity has been proposed as an alternative distance indicator for oldintermediate age stellar populations (Pulone 1992), while the ratio $N_{\mathrm{AGB}} / N_{\mathrm{HB}}$ is an excellent tool for investigating the efficiency of mixing processes during the HB phase (Buonanno et al. 1985), being HB lifetimes extremely sensitive to the extension of the semiconvective region in the stellar core.

In the two next sections we will first discuss the differences between "old" and "new" models, testing the most updated theoretical scenario on selected highquality Color-Magnitude (CM) diagrams of galactic GCs. Section 4 will deal with an investigation on the uncertainties still existing in current theoretical models. Concluding remarks will close the paper. 


\section{Theoretical models}

Theoretical predictions concerning AGB stars in galactic globulars have been presented and discussed in a previous paper ("old" models) about a decade ago (Castellani et al. 1991, hereinafter CCP). According to a quite common procedure, the discussion was based on HB models as produced by Red Giant Branch (RGB) progenitors with an original mass of $0.8 M_{\odot}$, in the assumption that, for ages of the order of $10^{10}$ years, differences in ages play a minor role in defining the structure and the evolution of He-burning stars. On this basis it was shown that in clusters with a well populated red HB theory predicts the occurrence of a clump of AGB stars with a rather well defined low luminosity edge. Theoretical predictions were found in reasonable agreement with the two main observational parameters, as given by i) the luminosity of the AGB clump with respect to the $\mathrm{HB}$ and, ii) the number ratio of $\mathrm{AGB}$ to $\mathrm{HB}$ stars, the so-called $R_{2}$ parameter. However, since that time evolutionary models have been progressively updated, following the availability of new and, hopefully, better physics and - in particular - of better neutrino energy losses, equation of state, opacities and nuclear cross sections. Thus the problem arises if a good fitting is preserved even in recent models.

To address this question, Fig. 1 shows the time behaviour of the luminosity for a typical HB model through and beyond the phase of central He-burning, i.e., along both HB and AGB phases, as computed in CCP or with the updated theoretical scenario presented in CCDW ("new" models), which takes also into account the efficiency of element sedimentation in the RGB progenitors. The most evident difference is the decrease of the HB lifetime, already discussed in CCDW. Consequently, the ratio of lifetimes in the two evolutionary phases, and thus the predicted star number ratio, is significantly different, changing from $\tau(\mathrm{AGB}) / \tau(\mathrm{HB}) \sim 0.11$ to about 0.15 . As we will discuss later on in this paper, numerical experiments disclose that such differences in the He-burning lifetimes are largely due to both the decreased efficiency of the ${ }^{12} \mathrm{C}(\alpha, \gamma){ }^{16} \mathrm{O}$ nuclear reaction in the "new" models and to the change in radiative opacities. An additional, but secondary, contribution to the decrease of central Heburning lifetime follows the larger luminosity of the new ZAHB models. However the same figure shows that the luminosity of both HB and AGB clump is increased by quite a similar amount, so that the difference in luminosity between these two observables $\left(\Delta M_{V}(\mathrm{AGB}-\mathrm{HB})\right)$ as predicted in $\mathrm{CCP}$, does survive the change of the physical inputs.

\section{Observational tests}

Taking advantage of the new and improved CM diagrams appeared in last years, one can repeat the analysis already given in CCP to test the adequacy of the theoretical scenario. To this purpose, we used Castelli et al. (1997) model atmospheres to translate bolometric luminosities and

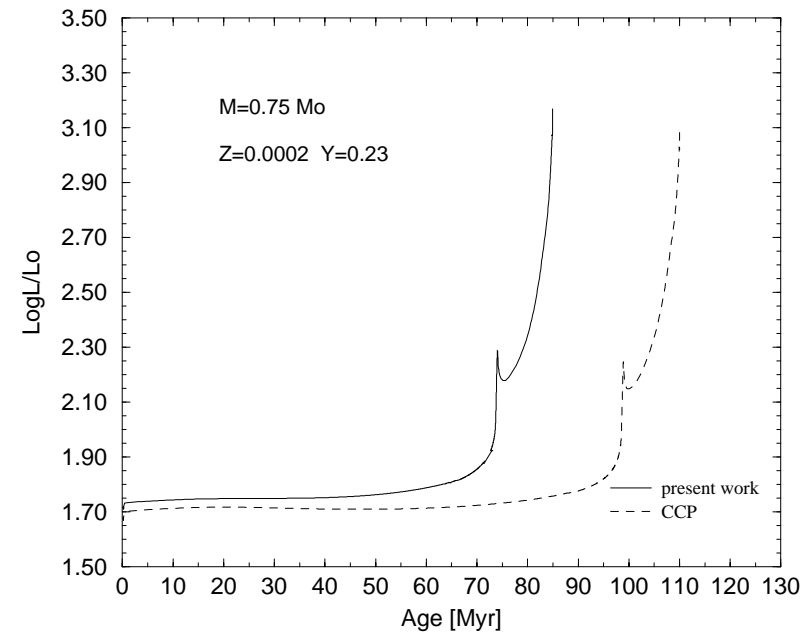

Fig. 1. Time behaviour of the surface luminosity during the central and shell He-burning phases for a model with the labelled mass and chemical composition, computed by adopting the most updated physics (solid line) or as in CCP (dashed line)

effective temperatures in the observational $\left(M_{V}, B-V\right)$ $\mathrm{CM}$ diagram, constraining the mixing length parameter by the requirement of reproducing the observed color of the AGB branches. In cool stars absolute visual magnitudes are indeed dependent on the adopted efficiency of the external convection which influences the effective temperature and, in turn, the bolometric correction; thus meaningful predictions for the luminosity of the AGB clump do require a suitable match of AGB colors.

Figure 2 shows the best fit of present $\mathrm{HB}$ and post-HB evolutionary tracks to the CCD CM diagram of M 5 presented by Sandquist et al. (1996). For the cluster metallicity we adopted from Sneden et al. (1992) $[\mathrm{Fe} / \mathrm{H}] \approx-1.17$ with $[\alpha / \mathrm{Fe}] \approx+0.2$. By adopting the relation given by Salaris et al. (1993), and from the value of $[\alpha / \mathrm{Fe}]$ one derives $Z=0.002([\mathrm{M} / \mathrm{H}] \approx-1.03)$. We also adopted $Y=$ 0.23 . As expected, by keeping as in CCP $E(B-V)=0.03$, in agreement also with the recent estimates by Sandquist et al. (1996), one obtains a reasonable fit, provided that the distance modulus estimated in CCP is increased by $\Delta(m-M)_{V}=0.09 \mathrm{mag}$ following the increased luminosity of the "new" models. This distance modulus appears in good agreement with the value provided by Sandquist et al. (1996, $\left.(m-M)_{V}=14.50 \pm 0.07 \mathrm{mag}\right)$. Sandquist et al. (1996) give for the observed number ratio $R_{2}=N_{\mathrm{AGB}} / N_{\mathrm{HB}}$ the value $0.169 \pm 0.06$. Within the uncertainty this observational value appears consistent both with $\mathrm{CCP}\left(\tau_{\mathrm{AGB}} / \tau_{\mathrm{HB}} \approx 0.11\right)$ and with present results $\left(\tau_{\mathrm{AGB}} / \tau_{\mathrm{HB}} \approx 0.15\right)$ even if the central value appears in better agreement with the new models. However, in the next section we will discuss the intrinsic weakness of such a theoretical prediction.

To test theoretical models at larger metallicities, the same fitting procedure has been applied to the highquality CM diagram for 47 Tuc (Sosin et al. 1996), as 
Table 1. Selected evolutionary quantities for Red Giant models with different metallicity, initial mass and He content (see text for more details). The age at the He flash is in Gyr

\begin{tabular}{ccccccc}
\hline \hline \multirow{2}{*}{$M\left(M_{\odot}\right)$} & $Z$ & $Y_{\mathrm{MS}}$ & $Y_{\text {pred }}$ & $\tau_{\text {flash }}(\mathrm{Gyr})$ & $M_{\mathrm{cHe}}\left(M_{\odot}\right)$ & $Y_{\mathrm{HB}}$ \\
\hline 0.80 & 0.0002 & 0.230 & 0.230 & 12.8 & 0.5148 & 0.2261 \\
0.80 & 0.002 & 0.230 & 0.234 & 15.4 & 0.5041 & 0.2305 \\
0.80 & 0.006 & 0.230 & 0.242 & 19.9 & 0.5001 & 0.2308 \\
0.80 & 0.006 & 0.242 & 0.242 & 18.3 & 0.4967 & 0.2457 \\
0.90 & 0.006 & 0.270 & 0.242 & 10.8 & 0.4892 & 0.2782 \\
0.95 & 0.006 & 0.230 & 0.242 & 11.0 & 0.4936 & 0.2420 \\
0.95 & 0.006 & 0.242 & 0.242 & 10.0 & 0.4933 & 0.2558 \\
\hline
\end{tabular}

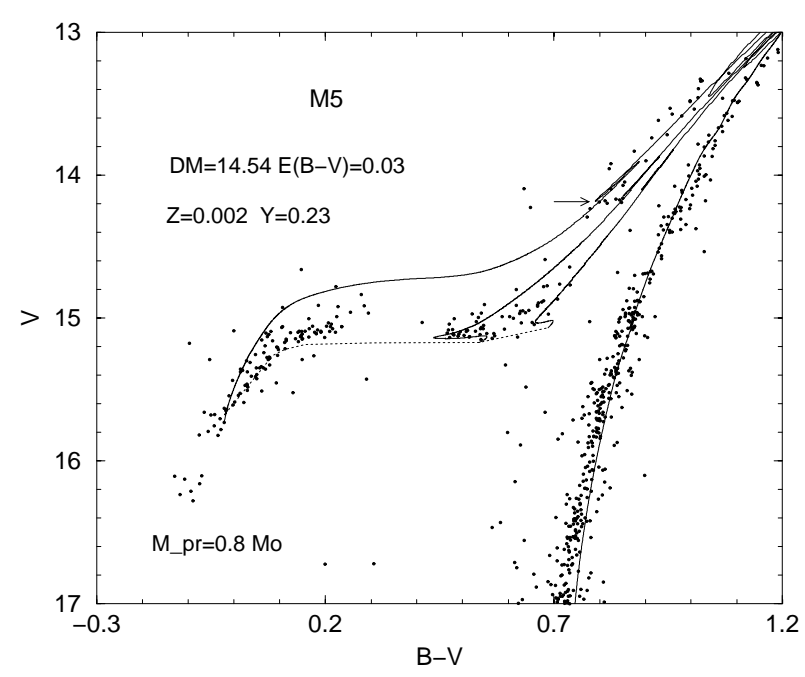

Fig. 2. The observed CM diagram of M 5 (Sandquist et al. 1996) with superimposed selected evolutionary tracks of $\mathrm{He}$ burning models with $0.8 M_{\odot}(Z=0.002, Y=0.23)$ RGB progenitors. The gap in the HB stellar distribution corresponds to the position of the instability strip. The theoretical ZAHB is shown as a dotted line. The horizontal arrow marks the predicted position of the low luminosity limit of the theoretical AGB clump

obtained with the WFPC2 camera of the Hubble Space Telescope. For the cluster metallicity, we adopted the spectroscopical measurement by Carretta \& Gratton (1997) $[\mathrm{Fe} / \mathrm{H}]=-0.7$ and an $\alpha$-element enhancement $[\alpha / \mathrm{Fe}]$ $\approx 0.2$ which corresponds to a mean between the estimates listed by Carney (1996) and by Salaris \& Cassisi (1996). Thus again from Salaris et al. (1993) one obtains $Z=0.006$. According to Schlegel et al. (1998) a cluster reddening $E(B-V)=0.03$ has been adopted.

With this choice Fig. 3 shows that the fitting of $\mathrm{HB}$ and AGB stars for 47 Tuc keeps being rather satisfactory. The derived distance modulus appears in agreement with the one obtained by Salaris \& Weiss (1998, $\left.(m-M)_{V}=13.42 \div 13.50 \mathrm{mag}\right)$ on theoretical basis, but smaller than the empirical value obtained by Gratton et al. $\left(1997,(m-M)_{V}=13.62 \pm 0.08 \mathrm{mag}\right)$ on the basis of Hipparcos subdwarfs.

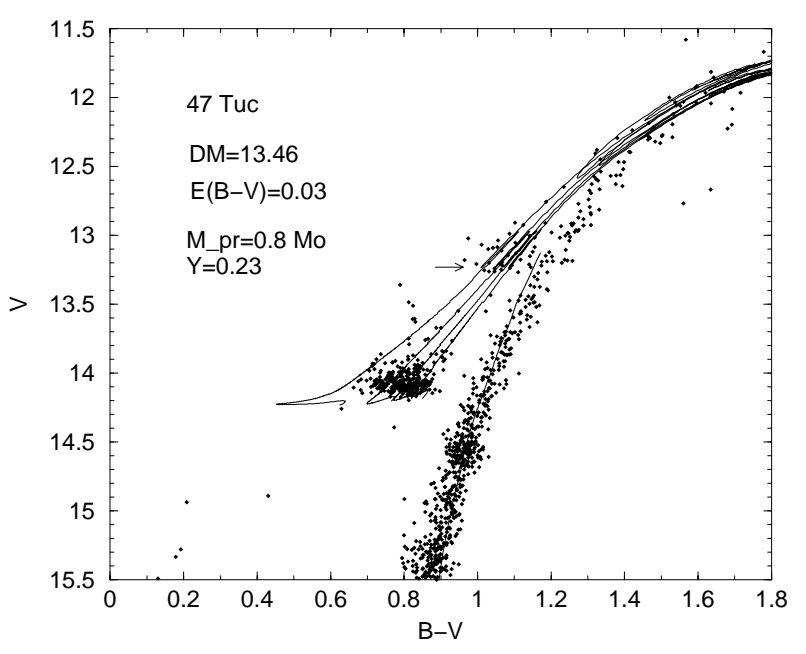

Fig. 3. The observed CM diagram of 47 Tuc (Sosin et al. 1996) with superimposed selected evolutionary tracks of He-burning models with a $0.80 M_{\odot}(Z=0.006, Y=0.23)$ RGB progenitor. The ZAHB models are fitted to the observed lower envelope of the HB distribution for the labelled assumptions on the distance modulus and reddening. The horizontal arrow marks the predicted lower envelope of the AGB clump

As already mentioned, in the previous fit of 47 Tuc we used the common procedure to adopt a $0.8 M_{\odot}(Y=$ $0.23)$ RGB progenitor. However, for moderately metal rich clusters this assumption is not completely satisfactory. To discuss this point Table 1 shows selected evolutionary parameters for Red Giant models with different metallicity, initial mass and He content. Left to right one finds: the mass of the RGB progenitor, the adopted values of $Z$ and initial Helium abundance $\left(Y_{\mathrm{MS}}\right)$, the value of $Y$ predicted ( $\left.Y_{\text {pred. }}\right)$ when accounting for a galactic Helium enrichment $\Delta Y / \Delta Z \approx 2.4$ (see, e.g., Pagel \& Portinari 1998; Castellani et al. 1999), the age at the He flash $\left(\tau_{\text {flash }}\right)$ together with the size $\left(M_{\mathrm{cHe}}\right)$ of the He core and the amount of surface $\mathrm{He}\left(Y_{\mathrm{HB}}\right)$ at this stage. One notices that the assumptions about the original $\mathrm{He}\left(Y_{\mathrm{MS}}=0.23\right)$ and the mass of the $\mathrm{RG}$ progenitor $\left(M=0.8 M_{\odot}\right)$ provide cluster ages which appear reasonably adequate for metal-poor globulars, with $Z \approx 0.0002$. However, when increasing the metallicity up to $Z=0.006$, the same assumptions would 


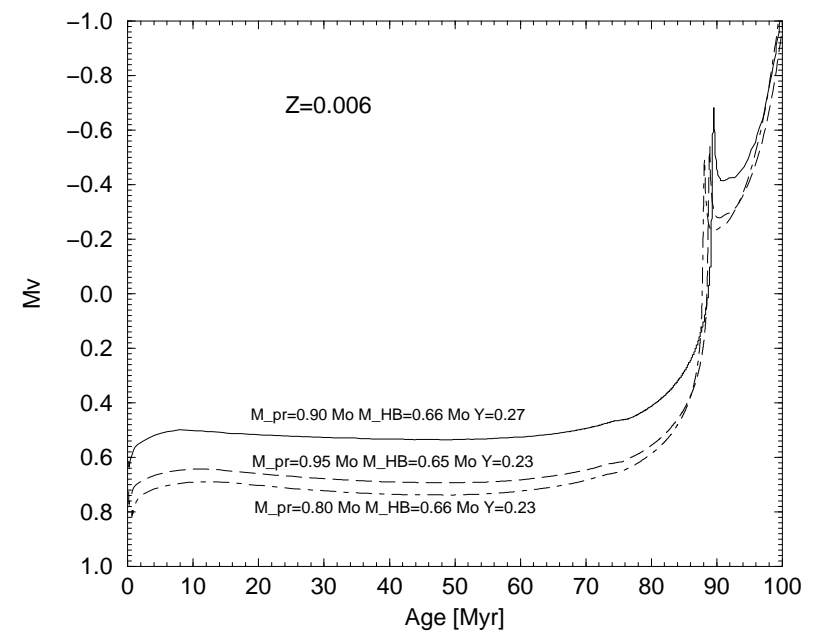

Fig. 4. Time behaviour of the absolute visual magnitude for He-burning models with selected RGB progenitors from the ZAHB to the AGB (see text)

imply an exceedingly large cluster age and an amount of original He not negligibly lower than expected by assuming a reasonable value for the galactic correlation between $Y$ and $Z$. The same table shows that an RGB progenitor of $0.95 M_{\odot}$ would give an age at the He flash in better agreement with present estimates for this cluster (Gratton et al. 1997; Salaris \& Weiss 1998).

Figure 4 shows the temporal behaviour of the visual magnitude for He burning models with $Z=0.006$ and selected RGB progenitors. The mass of the HB models has been chosen to approximately fit the center of the observational horizontal branch of 47 Tuc. As a relevant point, Fig. 4 shows that increasing the mass of the RGB progenitor (but adopting the same initial He abundance), the HB and AGB luminosity levels increase again by a rather similar amount. Thus one can easily predict that decreasing the age theory will fit the two He burning phases with the same accuracy, but with a distance modulus increased by $\Delta(m-M)_{V} \approx 0.06$. From the same figure one can estimate that passing from $Y=0.23$ to $Y=0.242$ one expects a further increase by about $0.04 \mathrm{mag}$. As a result, we drive the attention on the evidence that the commonly used assumptions for an RGB progenitor with $M=0.8 M_{\odot}$ and $Y=0.23$ can underestimate the cluster distance modulus by about $\Delta(m-M)_{V} \approx 0.1$. With such a correction, now one finds that our theoretical predictions appear in excellent agreement with the previous quoted estimate by Gratton et al. (1997).

However, regarding the helium content, Salaris \& Weiss (1998) noted that there are in literature some suggestions for an helium abundance of 47 Tuc close to the solar value. The same Fig. 4 shows a $0.66 M_{\odot} \mathrm{HB}$ model with a $0.9 M_{\odot} Y=0.27$ RGB progenitor. The age at the He flash is about $11 \mathrm{Gyr}$, in agreement with recent age estimates for 47 Tuc. One finds that now the HB luminosity is increased by about $0.23 \mathrm{mag}$ with respect to the model with $M=0.8 M_{\odot} Y=0.23$ progenitor whereas, even with this huge He variation, the $\Delta M_{V}(\mathrm{AGB}-\mathrm{HB})$ parameter is preserved with an accuracy of few hundredth of magnitude. Thus the observed $\Delta M_{V}$ (AGB-HB) cannot constrain the amount of original He. Nor the suggestion for an initial He abundance $Y \approx 0.27$ is ruled out by the luminosity of the RGB bump, as disclosed by numerical experiments.

Before closing this section one has to discuss briefly the reasons why $\Delta M_{V}(\mathrm{AGB}-\mathrm{HB})$ appears largely independent of variations in the assumed progenitor mass and/or original helium content. The latter point was already discussed by Pulone (1992), starting from the well known evidence that an increase in the original He content produces ZAHB models with smaller initial He-cores, but larger luminosities. The initial AGB luminosity is however also larger, since during $\mathrm{HB}$ evolution stars with higher helium abundance burn their hydrogen with larger rates, so that increasing the original He also increases the He-core at the He-exhaustion.

As for the behaviour of $\Delta M_{V}(\mathrm{AGB}-\mathrm{HB})$ with the progenitor mass, an increase of the RGB progenitor mass produces a slight decrease of the He core mass at the He ignition, and an increase of the envelope He abundance. In canonical models this is a consequence of a larger efficiency of the first dredge up. When element diffusion is taken into account this also arises from the evidence that larger masses have shorter evolutionary times and the efficiency of diffusion is thus reduced (see e.g. Proffit \& VandenBerg 1991; Castellani \& Degl'Innocenti 1999). According to the previous discussion, the competitive effects of these occurrences on the ZAHB and AGB clump luminosity, make almost constant the value of $\Delta M_{V}(\mathrm{AGB}-\mathrm{HB})$.

\section{Theoretical uncertainties}

In this section we will refer to the "new" evolutionary scenario to discuss the uncertainties affecting this as any other current theoretical prediction. We will separately discuss uncertainties produced by macroscopic mechanisms or by intrinsic uncertainties in the adopted input physics.

\subsection{Macroscopic mechanisms}

Any evaluation of HB and AGB models has to be based, implicitly or explicitly, on suitable assumptions about the efficiency of some macroscopic mechanisms. When dealing with low mass stars, one has to properly take into account: i) the amount of mass loss, ii) the efficiency of element sedimentation and, last but not least, iii) the amount of convective mixing in the stellar interior and, in this context, the debated occurrence of the so called "breathing pulses" (Sweigart \& Demarque 1972, 1973; Castellani et al. 1985; Sweigart 1990 and references therein). The amount of mass loss is constrained by the observed colour $\mathrm{HB}$ distribution, and it can be reasonably taken into account when fitting clusters CMD by reproducing the HB colour. On the contrary, the other two mechanisms have 


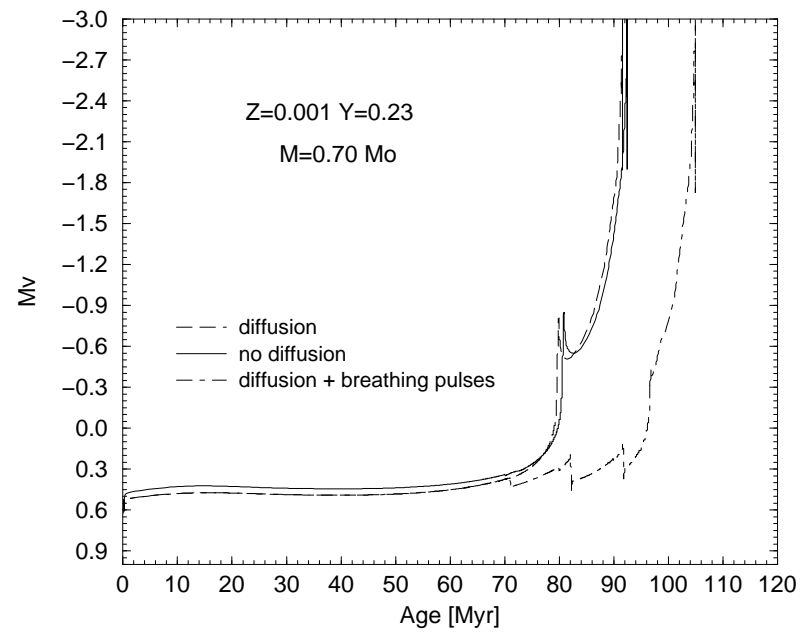

Fig. 5. Time behaviour of the absolute visual magnitude for a $0.7 M_{\odot}$ He-burning model from the ZAHB until the first thermal pulse by accounting (dashed line) or neglecting (solid line) atomic diffusion in the computation of the $0.8 M_{\odot} \mathrm{RGB}$ progenitor. The dot-dashed line corresponds to the $0.7 M_{\odot}$ He-burning model with diffusion when breathing pulses are allowed to occur during the central He-burning evolutionary phase

much more subtle effects, worth to be investigated in some details.

As for element diffusion, Fig. 5 compares the behaviour with time of the absolute visual magnitude for a typical HB model $\left(M=0.7 M_{\odot}\right)$ with or without microscopic diffusion in the H-burning progenitor. As already discussed (see, e.g. CCDW, Castellani \& Degl'Innocenti 1999) the difference is small: if diffusion is not taken into account the luminosity of both the HB and AGB models slightly increases by the same amount. Thus for no diffusion models the estimate of the distance modulus increases by about $\Delta M_{V} \approx 0.05 \mathrm{mag}$. In passing, we note that the evolutionary time of both the $\mathrm{HB}$ and AGB phases remains practically unchanged.

As shown in the same Fig. 5, this is not the case for the model with breathing pulses (which are suppressed in our reference "new" models), since the HB lifetime is increased by more than $20 \%$ whereas the AGB lifetime is decreased by more than $25 \%$. As a result the ratio $\tau_{\mathrm{AGB}} / \tau_{\mathrm{HB}}$ is dramatically reduced with respect to standard calculations. For models suitable for AGB stars in M 5 the inclusion of breathing pulses would produce a ratio $R_{2}=N_{\mathrm{AGB}} / N_{\mathrm{HB}} \sim 0.08$, well below the range of values allowed by observational constraints. In addition, Fig. 5 shows that if breathing pulses are at work, one expects a fainter and proportionally less populated AGB phase, with a less evident clumping of AGB stars at the bottom of the AGB branch. The occurrence of breathing pulses has been largely debated in the literature. Caputo et al. (1989) compared HB and RGB evolutionary lifetimes with observational data to conclude for the inefficiency of the pulses. One easily finds that present results clearly run

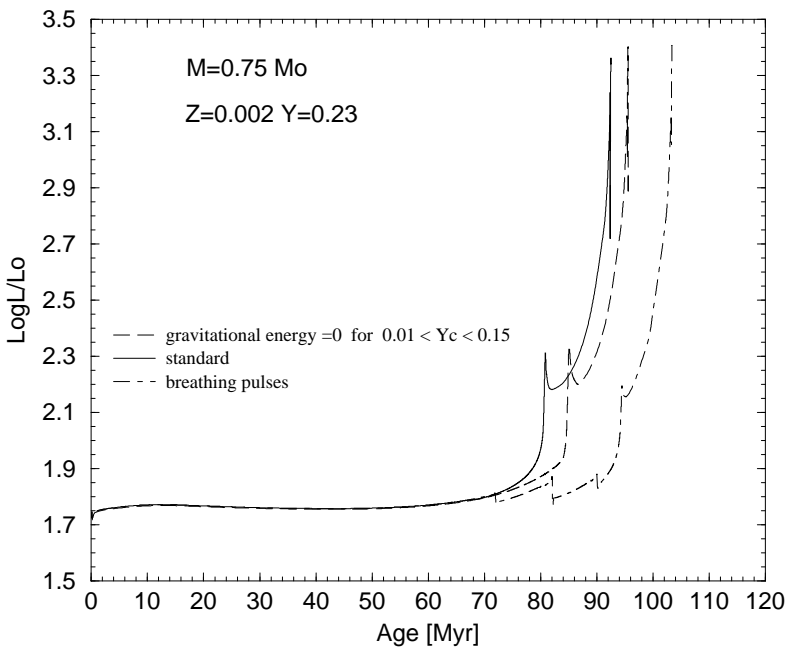

Fig. 6. Time behaviour of the surface luminosity for a $0.75 M_{\odot}$ He-burning model from the ZAHB until the first thermal pulse when breathing pulses are included (dot-dashed line). The figure also shows the same model when breathing pulses are dampened by adopting two different numerical algorithms: by preventing any He increase in the central convective core (solid line) or by neglecting the gravitational energy release during the phase of He exhaustion (dashed line, see text for more details). In all numerical experiments, microscopic diffusion of both $\mathrm{He}$ and heavy elements has been accounted for in the $0.8 M_{\odot}$ RGB progenitor

against observations, thus reinforcing the above quoted suggestion for the inefficiency of this phenomenon.

However, different algorithms to avoid the occurrence of breathing pulses in computing models have been presented in the literature. As discussed in Chieffi \& Straniero (1989), our evolutionary code just prevents any increase of He abundance in the central convective cores during the HB evolutionary phase, whereas Dorman \& Rood (1993) have shown that breathing pulses can be suppressed by neglecting the generation of gravitational energy during the core-helium exhaustion phase. Figure 6 shows the time behaviour of the luminosity of a $0.75 M_{\odot}$ He-burning model for these two alternative assumptions about breathing pulses suppression. One finds that neglecting the gravitational energy produces longer central He-burning lifetimes and slightly brighter AGB than our approach does. This because these models extend the semiconvection in a larger region than our standard models do. Even if the differences appear rather marginal, difficult to be detected within the current observational uncertainties, one should not forget that the way of suppressing the breathing pulses alone affects the theoretical models with an uncertainty in the HB lifetime of the order of $5 \%$, and of about $0.08 \mathrm{mag}$ in the absolute magnitude of the lower envelope of the AGB clump. In this context, we are actually reluctant to make a choice between the two quoted mechanisms. As a matter of fact, if our approach is rather crude, it is also true that gravitational energy is at the work in real stars. Thus the above quoted uncertainties appear to us unavoidables. 
Table 2. Selected results for a $0.75 M_{\odot}$ He-burning model with a $0.8 M_{\odot}$ RGB progenitor and metallicity $Z=0.0002$, under different assumptions about the adopted physical inputs. All the evolutionary lifetimes are in $10^{6}$ yrs

\begin{tabular}{|c|c|c|c|c|c|c|c|c|c|c|}
\hline & $\tau_{0.1}$ & $\tau_{\mathrm{HB}}$ & $\tau_{\mathrm{AGB}}$ & $\tau_{\mathrm{AGB}} / \tau_{\mathrm{HB}}$ & $\log \left(L / L_{\odot}\right)^{\mathrm{AGB}}$ & $M_{\mathrm{CO}}^{\mathrm{He}}\left(M_{\odot}\right)$ & $M_{\mathrm{CO}}^{\mathrm{AGB}}\left(M_{\odot}\right)$ & $X_{\mathrm{C}}$ & $X_{\mathrm{O}}$ & $X_{\mathrm{C}} / X_{\mathrm{O}}$ \\
\hline reference & 65.3 & 73.6 & 11.30 & 0.154 & 2.178 & 0.215 & 0.505 & 0.514 & 0.486 & 1.058 \\
\hline old cross sections & 72.4 & 81.4 & 10.95 & 0.134 & 2.173 & 0.217 & 0.505 & 0.240 & 0.760 & 0.316 \\
\hline EOS Straniero 1988 & 73.6 & 83.5 & 11.02 & 0.132 & 2.146 & 0.206 & 0.499 & 0.237 & 0.763 & 0.311 \\
\hline LAOL opacity & 80.9 & 90.9 & 10.97 & 0.121 & 2.148 & 0.221 & 0.506 & 0.230 & 0.769 & 0.299 \\
\hline old plasma neutrinos & 80.9 & 90.8 & 11.00 & 0.121 & 2.150 & 0.216 & 0.505 & 0.230 & 0.770 & 0.299 \\
\hline $\mathrm{CCP}$ & 87.4 & 98.4 & 11.00 & 0.112 & 2.148 & 0.213 & 0.495 & 0.225 & 0.775 & 0.290 \\
\hline
\end{tabular}

\subsection{Uncertainties in input physics}

In the previous Fig. 1 we have already shown the not negligible differences between present and CCP results, as produced by updating the physical inputs. To discuss these differences in more details we report in Table 2 selected quantities depicting evolutionary predictions for a $0.75 M_{\odot}$ He-burning model $(Z=0.0002, Y=0.23)$ under various assumptions about the input physics. Top to bottom the first line gives selected results from the present "new" reference scenario (with element diffusion) and, below, predictions for the same model but with progressive variations in the input physics eventually reproducing the input of CCP models. In all cases, but the "CCP" one, the helium core mass and the envelope helium abundance in the HB phase are those of the "new" reference model, that is the changes in the physical inputs are applied only to the HB evolution. The variations in the physics of the reference model run, top to bottom, as follows: i) nuclear cross sections from Caughlan \& Fowler (1988) to Caughlan et al. $(1985)$, ii) as in previous model but with the Equation of State of Straniero (1988) instead of the OPAL EOS (Rogers 1994; Rogers et al. 1996), iii) as in the previous model but with radiative opacity from Livermore opacity tables (Iglesias \& Rogers 1996) to Los Alamos opacities (Huebner et al. 1977), and electron conductivity from Itoh et al. (1983) to Hubbard \& Lampe (1969), iv) as in the previous model but passing from plasma neutrino energy losses of Haft et al. (1994) to those of Munakata et al. (1985). The last row gives the original CCP result as computed taking also into account the effects of the "old" physics on the RGB progenitor, that is by adopting as helium core mass and surface helium abundance the values by CCP.

Left to right the columns give: the time spent in the central He burning to reach a central He abundance $Y_{\mathrm{c}}=$ 0.1 (corresponding to the onset of breathing pulses, which are anyway suppressed), the total He-burning lifetime $\tau_{\mathrm{HB}}$, the AGB lifetime ( $\tau_{\mathrm{AGB}}$, from the exhaustion of central $\mathrm{He}$ to the onset of thermal pulses), the ratio $\tau_{\mathrm{AGB}} / \tau_{\mathrm{HB}}$, the bottom luminosity of the AGB clump, the mass of the CO core at the He exhaustion and at the first thermal pulse and, finally, the fractional abundance by mass of $\mathrm{C}$ and $\mathrm{O}$ in the $\mathrm{CO}$ core at the central He exaustion and the ratio of these two quantities.
One easily notices that the He-burning lifetime is mainly influenced by nuclear cross sections and by radiative opacity, which both affect the lifetime by about $9-10 \%$. As for nuclear cross sections, the main variation is due to the increase by about a factor 2 of the ${ }^{12} \mathrm{C}+\alpha$ cross section from Caughlan \& Fowler (1988) to Caughlan et al. (1985). The opacity effect is almost completely due to variations in carbon-oxygen opacity, which affects the opacity in the stellar core. One should also note the dramatic effect of the variation of ${ }^{12} \mathrm{C}+\alpha$ nuclear cross section on the $\mathrm{C} / \mathrm{O}$ ratio in the core, a quantity which is governing the subsequent white dwarf evolution (see e.g. D'Antona \& Mazzitelli 1990; Wood 1992; Salaris et al. 1997). Bearing in mind that a realistic estimate of current uncertainties affecting this cross section is as large as a factor of two (see Caughlan \& Fowler 1988; Buchmann 1996; Angulo et al. 1999), more precise measurements of this cross section at energies of astrophysical interest appear of great relevance.

\section{Conclusions}

In this paper we found that the "new" theoretical scenario arising from stellar models with updated physical inputs, appears able to account for the main observational constraints on GC AGB stars. However, we insist on the evidence that no theoretical result can be taken at its face value, because of still existing theoretical uncertainties. In this context we critically discussed the indetermination due either to the efficiency of some macroscopic mechanisms, like atomic diffusion and breathing pulses, or to the uncertainties on the input physics (equation of state, opacity, nuclear cross sections etc.). While the efficiency of atomic diffusion has little influence on He-burning models, the occurrence of breathing pulses is ruled out by the comparison between theory and observation, confirming the conclusion reached by Caputo et al. (1989) in the frame of the "old" theoretical scenario.

Regarding the physical inputs, the main uncertainty still present in He-burning models is given by the ${ }^{12} \mathrm{C}(\alpha, \gamma){ }^{16} \mathrm{O}$ cross section, which influences the He burning lifetimes and the $\mathrm{C} / \mathrm{O}$ ratio in the carbon-oxygen core, with relevant consequences on the final cooling of white dwarfs. Since this nuclear reaction rate has a strong effect on the predicted central He burning lifetimes, it affects also the evaluation of the initial He abundance in galactic 
GCs via the $R$ parameter, i.e., the ratio between the HB stars and the RGB ones brighter than the HB (see e.g. the discussion in CCDW and in Zoccali et al. 2000). On the contrary, data in Table 2 show that AGB lifetimes appear marginally affected by this uncertainty; therefore one could be tempted to use the ratio $N_{\mathrm{AGB}} / N_{\mathrm{HB}}$ as an indicator of efficiency of the ${ }^{12} \mathrm{C}+\alpha$ reaction. However, as discussed in the previous section, the extension of the mixed core of He-burning stars affects the value of $N_{\mathrm{AGB}} / N_{\mathrm{HB}}$ too, so that a comparison with a given CMD can only tell us if the combination of mixing prescription plus the adopted ${ }^{12} \mathrm{C}+\alpha$ reaction rate are consistent with observations. We have already shown that, at least for M 5, observational data are in agreement with the combination of the new reaction rate plus semiconvection without breathing pulses.

However, CCDW have already shown that current theoretical predictions for the $R$ parameter, as obtained by using the same input physics adopted in the present work, provide an unrealistically large value for the original He abundance in galactic GCs. This unpleasant situation could be clarified only by reducing the uncertainties related to the adopted physical inputs.

To summarize, the cross section for ${ }^{12} \mathrm{C}+\alpha$ reaction, the amount of central mixing in He burning stars, the evaluation of the original $\mathrm{He}$ in globular cluster stars and the ratio $N_{\mathrm{AGB}} / N_{\mathrm{HB}}$ are strongly connected. In this context, better evaluations of one or more out of the quoted quantities would be of great relevance to assess the problem on a more firm basis.

Acknowledgements. It is a pleasure to acknowledge G. Bono for a careful reading of the manuscript. We thank the anonymous referee for useful comments.

\section{References}

Angulo, C., Arnould, M., Rayet, M., et al. (NACRE collaboration) 1999, Nuclear Phys. A, 656, 3

Buchmann, L. 1996, ApJ, 468, L127

Buonanno, R., Corsi, C. E., \& Fusi Pecci, F. 1985, A\&A, 145, 97

Caputo, F., Castellani, V., Chieffi, A., Pulone, L., \& Tornambe, A. 1989, ApJ, 340, 241

Carney, B. W. 1996, PASP, 108, 900

Carretta, E., \& Gratton, R. G. 1997, A\&AS, 121, 95

Cassisi, S., Castellani, V., Degl'Innocenti, S., \& Weiss, A. 1998, A\&AS, 129, 267 (CCDW)

Cassisi, S., Castellani, V., Degl'Innocenti, S., Salaris, M., \& Weiss, A. 1999, A\&AS, 134, 103

Castellani, V., \& Degl'Innocenti, S. 1999, A\&A, 344, 97

Castellani, V., Chieffi, A., Pulone, L., \& Tornambe, A. 1985, ApJ, 296, 204
Castellani, V., Chieffi, S., \& Pulone, L. 1991, ApJS, 76, 911 (CCP)

Castellani, V., Degl'Innocenti, S., \& Marconi, M. 1999, A\&A, 349,834

Castelli, F., Gratton, R. G., \& Kurucz, R. 1997, A\&A, 318, 841

Caughlan, G. R., \& Fowler, W. A. 1988, Atom. Data Nucl. Data Tables, 40, 283

Caughlan, G. R., Fowler, W. A., Harris, M. J., \& Zimmerman, B. A. 1985, Atom. Data Nucl. Data Tables, 32, 197

Chaboyer, B. 1995, ApJ, 444, L9

Chieffi, A., \& Straniero, O. 1989, ApJS, 71, 47

D'Antona, F., \& Mazzitelli, I. 1990, ARA\&A, 28, 139

Dorman, B., \& Rood, R. T. 1993, ApJ, 409, 387

Gratton, R. G., Fusi Pecci, F., Carretta, E., et al. 1997, ApJ, 491, 749

Haft, M., Raffelt, G., \& Weiss, A. 1994, ApJ, 425, 222

Hubbard, W. B., \& Lampe, M. 1969, ApJS, 18, 297

Huebner, W. F., Merts, A. L., Magee, N. H., \& Argo, M. F. 1977, Los Alamos Sci. Lab. Rept., LA-6760-M

Iglesias, C. A., \& Rogers, F. J. 1996, ApJ, 464, 943

Itoh, N., Mitake, S., Iyetomi, H., \& Ichimaru, S. 1983, ApJ, 273,774

Munakata, H., Kohyama, Y., \& Itoh, N. 1985, ApJ, 296, 197

Pagel, B. E. J., \& Portinari, L. 1998, MNRAS, 298, 747

Proffitt, C. R., \& VandenBerg, D. A. 1991, ApJS, 77, 473

Pulone, L. 1992, Mem. SAIt, 63, 485

Rogers, F. J. 1994, in The equation of state in astrophysics, IUA Colloq. 147, ed. G. Chabrier, \& E. L. Schatzman (Cambridge University Press, Cambridge), 16

Rogers, F. J., Swenson, F. J., \& Iglesias, C. A. 1996, ApJ, 456, 902

Salaris, M., \& Cassisi, S. 1996, A\&A, 305, 858

Salaris, M., \& Weiss, A. 1998, A\&A, 335, 943

Salaris, M., Chieffi, A., \& Straniero, O. 1993, ApJ, 414, 580

Salaris, M., Dominguez, I., García-Berro, E., et al. 1997, ApJ, 486,413

Sandquist, E. L., Bolte, M., Stetson, P. B., \& Hesser, J. E. 1996, ApJ, 470, 910

Schlegel, D. J., Finkbeiner, D. P., \& Davis, M. 1998, ApJ, 500, 525

Sneden, C., Kraft, R. P., Prosser, C. F., \& Langer, G. E. 1992, AJ, 104, 2121

Sosin, C., Piotto, G., Djorgovski, S. G., et al. 1996, Proceeding of the workshop "Stellar Ecology", Marciana Marina, Italy, ed. R. D. Rood, \& A. Renzini, 92

Straniero, O. 1988, A\&AS, 76, 157

Sweigart, A. 1990, in Confrontation between stellar pulsation and evolution; Proceedings of the Conference, Bologna, Italy, May 28-31, San Francisco, CA, Astronomical Society of the Pacific 1990, 1

Sweigart, A. V., \& Demarque, P. 1972, A\&A, 20, 445

Sweigart, A. V., \& Demarque, P. 1973, in Variable Stars in Globular Clusters, D. Fernie (Dordrecht: Reidel), 221

Wood, M. A. 1992, ApJ, 386, 539

Zoccali, M., Cassisi, S., Bono, G., et al. 2000, ApJ, 538, 289 